\title{
The effects of different types of media on in vitro maturation outcomes of human germinal vesicle oocytes retrieved in intracytoplasmic sperm injection cycles
}

\author{
Farzaneh Fesahat ${ }^{1,2}$, Razieh Dehghani Firouzabadi ${ }^{2}$, Azita Faramarzi ${ }^{2}$, Mohammad Ali Khalilii \\ 'Department of Genetics, Shahid Sadoughi University of Medical Sciences, Yazd; ${ }^{2}$ Research and Clinical Center for Infertility, Shahid Sadoughi University \\ of Medical Sciences, Yazd, Iran
}

Objective: Optimizing in vitro maturation (IVM) media to achieve better outcomes has been a matter of interest in recent years. The aim of this prospective clinical trial was to investigate the effects of different media on the IVM outcomes of immature oocytes at the germinal vesicle (GV) stage.

Methods: A total of $400 \mathrm{immature}$ oocytes at the GV stage with normal morphology were retrieved from 320 infertile women aged $31 \pm 4.63$ years during stimulated intracytoplasmic sperm injection (ICSI) cycles. They were divided into groups of homemade IVM medium (I, $n=100)$, cleavage medium (II, $n=100)$, blastocyst medium (III, $n=100)$, and Sage IVM medium (IV, $n=100$ ) and cultured for 24 to 48 hours at $37^{\circ} \mathrm{C}$. ICSI was performed, and the rates of fertilization and embryo formation were compared across the four groups.

Results: In the 400 retrieved GV oocytes, the total maturation rates showed significant differences in groups I to IV $(55 \%, 53 \%, 78 \%$, and $68 \%$, respectively, $p<0.001)$. However, there were no significant differences in the fertilization, embryo formation, or arrest rates of metaphase II oocytes across these groups. In all groups, GV maturation was mostly completed after 24 hours, with fewer oocytes requiring 48 hours to mature $(p<0.01)$. Moreover, the rate of high-quality embryos was higher in group IV than in the other groups $(p=0.01)$.

Conclusion: The quality of the IVM medium was found to affect clinical IVM outcomes. Additionally, blastocyst medium may be a good choice in IVM/ICSI cycles as an alternative IVM medium.

Keywords: Embryo; Germinal vesicle; In vitro maturation; Intracytoplasmic sperm injections; Media

\section{Introduction}

In vitro maturation (IVM) of oocytes has the potential to offer a safe and cost-effective source of large numbers of embryos for in vitro fer-

Received: Jan 25, 2017 · Revised: Apr 3, 2017 · Accepted: May 17, 2017 Corresponding author: Mohammad Ali Khalili

Research and Clinical Center for Infertility, Shahid Sadoughi University of Medical Sciences, Bouali Ave, Safaiyeh, Yazd 8916877391, Iran

Tel: +98-3538247085-6 Fax:+98-3538247087 E-mail: khalili59@hotmail.com

*This article was adapted from the PhD thesis of Farzaneh Fesahat, which was financially supported by the Yazd Research and Clinical Center for Infertility.

This is an Open Access article distributed under the terms of the Creative Commons Attribution Non-Commercial License (http://creativecommons.org/licenses/by-nc/4.0/) which permits unrestricted non-commercial use, distribution, and reproduction in any medium, provided the original work is properly cited. tilization (IVF) treatment. It does not rely on superovulation or synchronization, and poses virtually no risk of ovarian hyperstimulation syndrome [1,2]. Additionally, IVM involves preparing a controlled environment for the production of embryos for manipulation in fields such as transgenics, cloning, and stem cells for research, even from stimulated IVF cycles [1,2]. It has been shown that after ovarian stimulation, approximately $15 \%$ of the retrieved oocytes are immature, at the germinal vesicle (GV) and GV breakdown (maturity [MI]) stages $[3,4]$. IVM may increase the chance that patients will utilize their oocytes to the greatest extent possible in an IVF program. For IVM success, the choice of a suitable culture medium plays an important role, since low oocyte maturation and embryo development rates following IVM are considered major complications [5-7]. Various media for 
IVM have been proposed in different clinical or research studies, including human tubal fluid (HTF) [8], culture medium 199 [9,10], and blastocyst medium [10]. Additionally, there are two commercially available IVM media, MediCult (Origio, Jyllinge, Denmark) and Sage IVM media (Cooper Surgical, Trumbull, CT, USA), which are often used with reasonable fertilization, implantation, and pregnancy rates [11]. However, applying these specially formulated commercial media has several disadvantages, such as a limited shelf life and the fact that they are more costly than standard culture media for assisted IVF centers where IVM services are not routinely performed. A recent study showed that blastocyst medium was suitable for the IVM of human immature oocytes, based on a comparison with Sage IVM medium in unstimulated IVF cycles [12]. Therefore, the aim of this study was to compare the efficacy of four types of media (homemade IVM medium, cleavage medium, blastocyst medium, and Sage IVM medium) in stimulated intracytoplasmic sperm injection (ICSI) cycles, as assessed by the rates of oocyte maturation, fertilization, and high-quality embryo formation.

\section{Methods}

\section{Patients}

A total of 320 infertile women aged $31 \pm 4.63$ years who had not experienced previous IVF failure were included in this study; women aged $\geq 35$ years, poor responders, and patients with a history of previous IVF failure were excluded. The oocytes that were used in each group were matched according to normal appearance and female age. Only morphologically normal GV oocytes were included. Oocytes were excluded due to either cytoplasmic or extracytoplasmic abnormalities such as shape, dark cytoplasm, diffuse granulation, perivitelline space, central cytoplasmic granulation, or being at another stage of Ml. The patients were stimulated with a standard gonadotropin-releasing hormone antagonist protocol [13]. Each ICSI cycle with morphologically immature GV oocytes was included in this study, and the oocytes were randomly divided into four groups. The randomization of oocytes into groups was performed using random number tables. Immature oocytes were used for research with consent from the patients admitted to our institute. This study was approved by the ethics committee of the Research and Clinical Center for Infertility, Yazd, Iran. Human chorionic gonadotropin (10,000 IU; Ferring, Kiel, Germany) was injected 36 hours before oocyte collection. The collected oocytes were cultured in IVF media (Vitrolife, Kungsbacka, Sweden) and placed in a $6 \% \mathrm{CO}_{2}$ incubator $\left(37^{\circ} \mathrm{C}\right)$ for 3 hours. Cumulus cells were removed enzymatically with $80 \mathrm{IU} / \mathrm{mL}$ of hyaluronidase (Sigma, St. Louis, MO, USA) and mechanically using glass pipettes. Denuded oocytes were assessed for nuclear maturity under a stereomicroscope (Olympus Co., Tokyo, Japan).

\section{IVM and ICSI protocols}

In this prospective experimental study, normal $400 \mathrm{GV}$ oocytes were retrieved from stimulated ICSI cycles. They were washed twice with G-Mops-V1 (Vitrolife), and were divided into the following four groups: homemade IVM medium (I, $\mathrm{n}=100)$, cleavage medium (II, $\mathrm{n}=100$ ), blastocyst medium (III, $\mathrm{n}=100$ ) and Sage IVM medium (IV, $\mathrm{n}=100$ ). Immature oocytes were cultured in $25-\mu \mathrm{L}$ drops in all maturation media, supplemented with $75 \mathrm{IU} / \mathrm{L}$ of human menopausal gonadotropin (IVF-M; LG Life Sciences, Iksan, Korea), and cultured for 24 to 48 hours at $37^{\circ} \mathrm{C}$ in $5 \% \mathrm{O}_{2}, 6 \% \mathrm{CO}_{2}$, and $90 \%$ air with high humidity under paraffin oil (MediCult, Origio).

The homemade IVM medium consisted of Ham's F10 medium (Biochrom, Berlin, Germany) supplemented with 0.75 IU of luteinizing hormone and $0.75 \mathrm{IU}$ of follicle-stimulating hormone (Ferring) with $40 \%$ human follicular fluid [14]. Briefly, human follicular fluid was centrifuged at 3,500 rpm for 10 minutes to separate the blood and granulosa cells. After inactivation at $56^{\circ} \mathrm{C}$ for 30 minutes, the clear follicular fluid was filtered with a $0.22-\mu \mathrm{m}$ filter and was used for media preparation. The oocytes were assessed for maturity after 24 and 48 hours at $37^{\circ} \mathrm{C}$ in a humidified atmosphere of $6 \% \mathrm{CO}_{2}$ in air. Oocyte maturation was assessed by the presence of the first polar body under an inverted microscope (Nikon, Tokyo, Japan) [15]. ICSI was performed for in vitro matured oocytes in order to compare the rates of fertilization and embryo formation across the four groups. After ICSI, oocytes were cultured in 20- $\mu \mathrm{L}$ drops of cleavage medium (Cook Medical, Limerick, Ireland) in groups I, II, and III and in Sage embryo maintenance medium in group IV in a culture dish covered with paraffin oil. Fertilization was checked 18 hours after ICSI and calculated in each group. Fertilized oocytes were then cultured in maintenance or cleavage medium for 72 hours, and embryo arrest and the presence of high-quality embryos were assessed according to the Hill embryo grading system [16]. In order to limit the extent of technical bias, ICSI was performed by a single expert embryologist.

\section{Statistical analysis}

The data were categorical and presented as frequencies and percentages. When more than $25 \%$ of table cells had a frequency less than $5 \%$, the chi-square test was used for statistical analysis. In tables where the data frequency was smaller, the Fisher exact test, with less power, was used. Comparisons of frequency data between groups, such as the fertilization and developmental rates, were performed using the chisquare test in SPSS ver. 20.0 (IBM Corp., Armonk, NY, USA). The $p$-values $<0.05$ were considered to indicate statistical significance.

\section{Results}

A total of 449 immature oocytes were obtained from 320 stimulat- 
ed ICSI cycles in patients without previous IVF failure. According to the exclusion criteria, 49 oocytes (12.25\%) were excluded from the study, while 400 oocytes (87.75\%) with normal morphological criteria were included. Patients with primary infertility comprised $85 \%$ of the group, while $15 \%$ had secondary infertility. The etiology of infertility for the patients was male factor for $40 \%$, unknown for $27 \%$, female factor for $21 \%$, and both male and female factor for $12 \%$.

The total maturation rates of the $400 \mathrm{GV}$ oocytes showed significant differences in groups I to IV (55\%, 53\%, 78\%, and 68\%, respectively, $p<0.001)$; a higher percentage was present in group III (78\%) than in group IV, although this was not significant. Additionally, similar maturation rates were found for the oocytes in groups I and II with different incubation times (38\% vs. $43 \%$ in the 24 -hour group and $17 \%$ vs. $10 \%$ in the 48 -hour group) (Figure 1). In general, the total fertilization rates were similar in groups I and II (52.7\% and 56.6\%, respectively), whereas improved rates were found using blastocyst and Sage IVM media (group III, 69\%; group IV, 54.7\%). There were no significant differences in the total fertilization rates of IVM oocytes or the rate of abnormally fertilized oocytes (one pronucleus or multiple pronuclei) in IVM oocytes across the media groups ( $p=0.1$ and $p=0.9$, respectively).
There were no significant differences in the embryo formation or arrest rates among the fertilized oocytes across the media groups. However, the highest embryo formation rate was found in the group

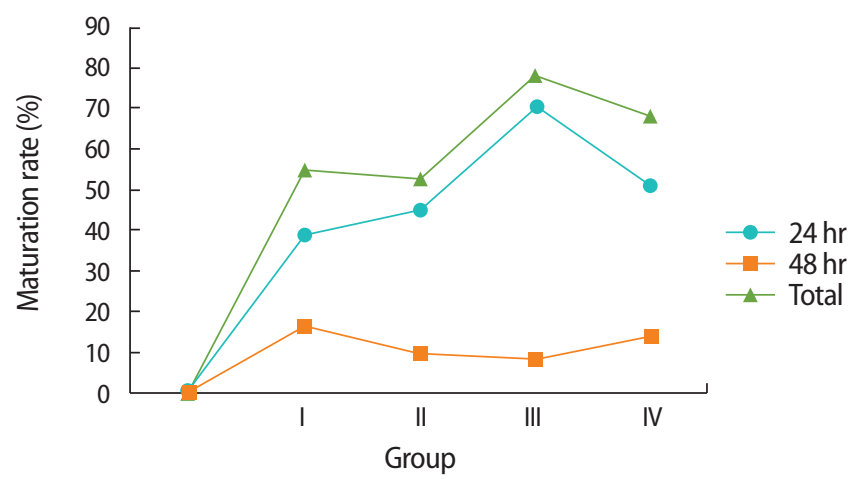

Figure 1. Nuclear maturation after 24 or 48 hours of culture in different in vitro maturation media. The germinal vesicle (GV) maturation rate was significantly higher after 24 hours of incubation than after 48 hours of incubation in each group $(p<0.01)$ and showed a significant difference at 48 hours compared to 24 hours of incubation in each group $(p<0.01)$; the GV maturation rate was significantly lower at 48 hours compared to the total time duration in each group ( $p$ <.01). Groups: I, homemade IVM medium; II, cleavage medium; III, blastocyst medium; IV, Sage IVM medium.

Table 1. Nuclear maturation, fertilization, embryo formation, and developmental status of GV oocytes after culture in different IVM media

\begin{tabular}{|c|c|c|c|c|c|}
\hline Variable & I & ॥ & III & IV & $p$-value \\
\hline Maturation, IVM oocytes/total GV oocytes & $55 / 100(55)$ & $53 / 100(53)$ & $78 / 100(78)$ & $68 / 100(68)$ & $\begin{aligned} &<0.001^{\mathrm{a})} \\
& 0.05^{\mathrm{b})} \\
& 0.001^{\mathrm{a}, \mathrm{c}} \\
&<0.001^{\mathrm{a}, \mathrm{d})}\end{aligned}$ \\
\hline Normal fertilized oocytes/total GV oocytes & $29 / 100(29)$ & $30 / 100(30)$ & $54 / 100(54)$ & $37 / 100(37)$ & $\begin{array}{l}0.001^{\mathrm{a})} \\
0.001^{\mathrm{c}} \\
\left.0.001^{\mathrm{d}}\right) \\
0.02^{\mathrm{b})}\end{array}$ \\
\hline Normal fertilized oocytes/IVM oocytes & $29 / 55(52.7)$ & $30 / 53(56.6)$ & $54 / 78(69)$ & $37 / 68(54.7)$ & 0.1 \\
\hline Abnormal fertilized oocytes/IVM oocytes & $3 / 55(5.4)$ & $3 / 53(5.7)$ & $3 / 78(3.9)$ & $2 / 68(2.9)$ & 0.9 \\
\hline Formed embryos/total GV oocytes & $27 / 100(27)$ & $26 / 100(26)$ & $47 / 100(47)$ & $35 / 100(35)$ & $\begin{array}{l}0.005^{\mathrm{a})} \\
0.002^{\mathrm{d})} \\
0.003^{\mathrm{c})} \\
0.08^{\mathrm{b})}\end{array}$ \\
\hline Formed embryos/IVM oocytes & $27 / 55(49)$ & $26 / 53(50)$ & $47 / 78(61)$ & $35 / 68(51.5)$ & 0.5 \\
\hline Formed embryos/fertilized oocytes & $27 / 29(93.1)$ & $26 / 30(86.7)$ & $47 / 54(87)$ & $35 / 37(94.3)$ & 0.3 \\
\hline Embryo arrest/fertilized oocytes & $4 / 29(13.6)$ & $3 / 30(10)$ & $5 / 54(9.5)$ & $2 / 37(5.4)$ & 0.7 \\
\hline High-quality embryos & $8 / 27(29.6)$ & $9 / 26(33.3)$ & $15 / 47(31)$ & $21 / 35(60.0)$ & $\begin{array}{l}0.01^{\mathrm{a})} \\
0.02^{\mathrm{e})} \\
0.01^{\mathrm{f})} \\
0.004^{\mathrm{b})}\end{array}$ \\
\hline
\end{tabular}

Groups: I, homemade IVM medium; II, cleavage medium; III, blastocyst medium; IV, Sage IVM medium. High-quality embryos were defined as grade A and B embryos. Values are presented as number (\%).

GV, germinal vesicle; IVM, in vitro maturation.

${ }^{a)}$ A significant difference was present between medium groups $(p<0.01)$; A significant difference was present between ${ }^{\text {b) }}$ group III and IV; ${ }^{c)}$ group I and III; ${ }^{d)}$ group II and III; e) group I and IV; f) group II and IV. 
treated with Sage IVM medium (Table 1). Most GV maturation had been completed after 24 hours (38\%, $43 \%, 70 \%$, and $53 \%$ in groups I-IV, respectively), instead of after 48 hours $(17 \%, 10 \%, 8 \%$, and $15 \%$ in groups I-IV, respectively) (Figure 1), although no significant differences in the fertilization and embryo formation rates were found according to the time to maturation in any group. Moreover, the rate of high-quality embryo formation (grade $A$ and grade $B$ ) was higher in group IV than in other groups $(p=0.01$ ) (Table 1). The results also showed that the embryos derived from oocytes cultured in homemade and cleavage media were of lower quality than those derived from oocytes cultured in blastocyst or Sage media, and were mostly categorized in grades $C$ and $D$.

\section{Discussion}

We compared four different culture media in terms of maturation, fertilization, and embryonic developmental competence in an IVM program during stimulated ICSI. The highest maturation rate of oocytes was found in blastocyst medium, regardless of the time to maturation. The optimal time for obtaining large amounts of IVM oocytes was after 24 hours. A longer incubation time of GV oocytes did not significantly improve the maturation rate. There is no consensus regarding the optimal maturation timing of oocytes in IVM cycles, and various approaches to timing have been reported in experimental studies $[12,17]$. However, a previous study showed that the rates of cleavage and blastocyst development in oocytes matured 48 hours after IVM $(72.2 \%, 19.0 \%)$ were significantly lower than in those that matured 24 hours after IVM $(91.5 \%, 50.4 \%)$ [17], which agrees with our results. That study suggested that oocytes that reach metaphase II faster in an IVM program have better embryonic developmental competence [17]. Furthermore, a study conducted in 2013 stated that in stimulated ICSI cycles, immature oocytes were cultured for 24 to 30 hours and oocytes were injected for further culturing after extrusion of the polar body [18].

The total fertilization rates were similar in groups I and II, but were higher when blastocyst and Sage IVM media were used. However, longer incubation did not significantly change the total fertilization rates in groups I to IV $(52.7 \%, 56.6 \%, 69 \%$, and $54.7 \%$, respectively). This may indicate that the higher fertilization rate in group III is still present when immature oocytes are incubated for a longer time in blastocyst medium.

Few studies have compared the efficacy of different IVM media on immature oocytes obtained from stimulated or non-stimulated cycles. Recently, Pongsuthirak et al. [12] and Pongsuthirak and Vutyavanich [19] compared MediCult IVM media (Origio) with Sage IVM media (Cooper Surgical), as well as blastocyst and Sage media, in two randomized controlled trials using immature human oocytes ob- tained during cesarean deliveries. They showed no differences $(p>0.05$ ) in maturation (65\% vs. $69 \%$ ) or fertilization (65.2\% vs. $69 \%$ ) between oocytes in the different media groups. Despite differences in the study design, such as using non-stimulated immature oocytes and an IVM duration of 36 hours, the results were compatible with the present findings regarding the maturation and fertilization rates obtained using blastocyst and Sage IVM media [12,19]. Moreover, other studies using various IVM media have reported maturation rates of $44 \%$ to $67 \%$ after 28 to 36 hours of in vitro culture, and fertilization rates of $40 \%$ to $77 \%[2,9,20]$. These studies reported rates of high-quality blastocyst formation of $45.9 \%$ to $46.6 \%$. In contrast, in this study, only embryo formation after checking for two pronuclei and the rate of high-quality embryos at the cleavage stage (day 3 ) were used as assessments of the endpoint. However, the cleavage rates of previous studies agreed with the embryo formation rates observed in the present study (Table 1). Lower maturation, fertilization, and embryo formation rates were observed in both homemade and cleavage media than in other media.

In this lab trial, all IVM media were supplemented with $0.75 \mathrm{IU}$ of luteinizing hormone and $0.75 \mathrm{IU}$ of follicle-stimulating hormone (Ferring), which may increase the oocyte maturation rate by promoting the production of meiosis-activating substances [21]. The cleavage and homemade media have plasma protein sources. However, these media lack compounds such as essential amino acids and monobasic anhydrous potassium phosphate when compared with blastocyst and Sage media, and these compounds may influence the process of oocyte maturation $[22,23]$. In a review article, Farsi et al. [18] compared the formulations of different media and found that taurine and calcium, as unique components of blastocyst medium, were likely valuable for IVM media. These substances were proposed to be useful because taurine and hypotaurine are important antioxidants that are present in gametes and in the embryonic environment in abundance. Additionally, blastocyst culture media containing calcium lactate may have positive effects on mitochondrial function, helping to ensure sufficient adenosine triphosphate production. Moreover, Moschini et al. [24] showed that commercial IVM media have no significant benefits for improving the maturation of oocytes obtained from stimulated cycles compared with standard growth media.

Some studies have used other basic or modified media for specific subcategories of patients. One study in patients with polycystic ovary syndrome (PCOS) found TCM-199 culture medium to be superior to HTF-supplemented medium for oocyte maturation, fertilization, and subsequent embryo development; they reported findings similar to those of the present study regarding the maturation rate $(56.9 \%$ vs. $55.5 \%)$, fertilization rate (39.4\% vs. $53 \%)$, and embryo quality ( $41.7 \%$ vs. $28.5 \%$ ). They concluded that HTF medium is not an appropriate 
medium for the maturation of oocytes obtained from PCOS patients in non-stimulated cycles [8]. As well, de Araujo et al. [8] reported that TCM 199 was better than HTF medium in the IVM of oocytes obtained from PCOS patients in terms of the maturation rate $(82 \%$ vs. $56.9 \%)$, fertilization rate ( $70 \%$ vs. $39.4 \%)$, and embryo quality $(81.3 \%$ vs. $41.7 \%$ ). In contrast, Kim et al. [10] demonstrated that TCM-199 culture medium was unsuitable in comparison with Sage and blastocyst media. It seems that these findings may refer to non-specific and basic formulated compositions of media for IVM purposes, and that such formulations are not capable of satisfying all the in vitro nuclear and cytoplasmic maturation needs of immature oocytes. The first step of the maturation of immature oocytes may be the main contributor to poor IVM outcomes in the next steps of fertilization and embryo formation in those groups, in contrast to groups III and IV in our study. This is supported by the observation that the embryo formation rates in groups I and II were reasonably successful in oocytes that had completed the cytoplasmic and nucleic maturation processes and had been successfully fertilized. Moreover, some investigations have demonstrated that plasma protein supplements can supply additional factors involved in the regulation of oocyte maturation [22].

Because of the diversity in the number of immature oocytes obtained from each ICSI cycle, randomization was performed based on oocytes per group, with one normal oocyte per group. Furthermore, a limitation of this study was the fact that we could not evaluate the clinical outcomes, because the immature oocytes retrieved from stimulated cycles are not usually used in patients and are therefore discarded. Further studies should be done to ascertain the implantation and pregnancy potential of these embryos. However, we tried to minimize errors by considering various aspects of study design, such as sample size calculation for a maturation rate of immature oocytes of at least 50\% using any kind of medium, the inclusion criteria for oocyte selection, and having a single expert perform ICSI. Moreover, for human IVM, definitive conclusions on the establishment of imprinting are currently not available [25]. A systematic review of animal models provides reassuring data on imprinting establishment in cultured oocytes. Nevertheless, detailed studies of how chromosomal status and genomic imprinting patterns may be associated with the effects of different culture media on IVM outcomes are needed. In conclusion, it seems that the quality of the IVM media affected clinical IVM outcomes. Additionally, blastocyst medium may be a good choice in IVM/ICSI cycles as an alternative IVM medium.

\section{Conflict of interest}

No potential conflict of interest relevant to this article was reported.

\section{Acknowledgments}

The authors would like to thank all colleagues who helped with experiments and data collection.

\section{References}

1. Practice Committees of the American Society for Reproductive Medicine and the Society for Assisted Reproductive Technology. In vitro maturation: a committee opinion. Fertil Steril 2013;99: 663-6.

2. Gremeau AS, Andreadis N, Fatum M, Craig J, Turner K, McVeigh E, et al. In vitro maturation or in vitro fertilization for women with polycystic ovaries? A case-control study of 194 treatment cycles. Fertil Steril 2012;98:355-60.

3. Al-Hasani S, Ozmen B, Koutlaki N, Schoepper B, Diedrich K, Schultze-Mosgau A. Three years of routine vitrification of human zygotes: is it still fair to advocate slow-rate freezing? Reprod Biomed Online 2007;14:288-93.

4. Kuwayama M, Vajta G, Kato O, Leibo SP. Highly efficient vitrification method for cryopreservation of human oocytes. Reprod Biomed Online 2005;11:300-8.

5. Combelles CM, Chateau G. The use of immature oocytes in the fertility preservation of cancer patients: current promises and challenges. Int J Dev Biol 2012;56:919-29.

6. Shu Y, Gebhardt J, Watt J, Lyon J, Dasig D, Behr B. Fertilization, embryo development, and clinical outcome of immature oocytes from stimulated intracytoplasmic sperm injection cycles. Fertil Steril 2007;87:1022-7.

7. Blanco MR, Demyda S, Moreno Millan M, Genero E. Developmental competence of in vivo and in vitro matured oocytes: a review. Biotechnol Mol Biol Rev 2011;6:155-65.

8. de Araujo CH, Nogueira D, de Araujo MC, Martins Wde P, Ferriani RA, dos Reis RM. Supplemented tissue culture medium 199 is a better medium for in vitro maturation of oocytes from women with polycystic ovary syndrome women than human tubal fluid. Fertil Steril 2009;91:509-13.

9. Filali M, Hesters L, Fanchin R, Tachdjian G, Frydman R, Frydman N. Retrospective comparison of two media for invitro maturation of oocytes. Reprod Biomed Online 2008;16:250-6.

10. Kim M, Hong SJ, Lee JH, Min CK, Hwang KJ, Park RW. Comparison of in vitro maturation media of immature oocytes: the effectiveness of blastocyst culture media. Fertil Steril 2011;95:554-7.

11. Son WY, Chung JT, Demirtas E, Holzer H, Sylvestre C, Buckett W, et al. Comparison of in-vitro maturation cycles with and without in-vivo matured oocytes retrieved. Reprod Biomed Online 2008; 17:59-67. 
12. Pongsuthirak $P$, Songveeratham $S$, Vutyavanich T. Comparison of blastocyst and Sage media for in vitro maturation of human immature oocytes. Reprod Sci 2015;22:343-6.

13. Eftekhar M, Aflatoonian A, Mohammadian F, Eftekhar T. Adjuvant growth hormone therapy in antagonist protocol in poor responders undergoing assisted reproductive technology. Arch Gynecol Obstet 2013;287:1017-21.

14. Shahedi A, Hosseini A, Khalili MA, Norouzian M, Salehi M, Piriaei $A$, et al. The effect of vitrification on ultrastructure of human in vitro matured germinal vesicle oocytes. Eur J Obstet Gynecol Reprod Biol 2013;167:69-75.

15. Safian F, Khalili MA, Karimi-Zarchi M, Mohsenzadeh M, Ashourzadeh S, Omidi M. Developmental competence of immature oocytes aspirated from antral follicles in patients with gynecological diseases. Iran J Reprod Med 2015;13:507-12.

16. Hill GA, Freeman M, Bastias MC, Rogers BJ, Herbert CM 3rd, Osteen KG, et al. The influence of oocyte maturity and embryo quality on pregnancy rate in a program for in vitro fertilizationembryo transfer. Fertil Steril 1989;52:801-6.

17. Son WY, Lee SY, Lim JH. Fertilization, cleavage and blastocyst development according to the maturation timing of oocytes in in vitro maturation cycles. Hum Reprod 2005;20:3204-7.

18. Farsi MM, Kamali N, Pourghasem M. Embryological aspects of oocyte in vitro maturation. Int J Mol Cell Med 2013;2:99-109.

19. Pongsuthirak P, Vutyavanich T. Comparison of Medicult and Sage media for in vitro maturation of immature oocytes obtained dur- ing cesarean deliveries. J Fertil In Vitro IVF Worldw Reprod Med Genet Stem Cell Biol 2015;3:136.

20. Gardner DK, Lane M, Spitzer A, Batt PA. Enhanced rates of cleavage and development for sheep zygotes cultured to the blastocyst stage in vitro in the absence of serum and somatic cells: amino acids, vitamins, and culturing embryos in groups stimulate development. Biol Reprod 1994;50:390-400.

21. Byskov AG, Yding Andersen C, Hossaini A, Guoliang X. Cumulus cells of oocyte-cumulus complexes secrete a meiosis-activating substance when stimulated with FSH. Mol Reprod Dev 1997;46: 296-305.

22. Meintjes M. Media composition: macromolecules and embryo growth. In: Smith GD, Swain JE, Pool TB, editors. Embryo culture: methods and protocols. Totowa: Humana Press; 2012. p. 107-27.

23. Sutton ML, Gilchrist RB, Thompson JG. Effects of in-vivo and invitro environments on the metabolism of the cumulus-oocyte complex and its influence on oocyte developmental capacity. Hum Reprod Update 2003;9:35-48.

24. Moschini RM, Chuang L, Poleshchuk F, Slifkin RE, Copperman AB, Barritt J. Commercially available enhanced in vitro maturation medium does not improve maturation of germinal vesicle and metaphase I oocytes in standard in vitro fertilization cases. Fertil Steril 2011;95:2645-7.

25. Anckaert E, De Rycke M, Smitz J. Culture of oocytes and risk of imprinting defects. Hum Reprod Update 2013;19:52-66. 